\title{
The naked truth: Positive, arousing distractors impair rapid target perception
}

\author{
Steven B. Most \\ University of Delaware, Newark, DE, USA \\ Stephen D. Smith, Amy B. Cooter, Bethany N. Levy, and \\ David H. Zald \\ Vanderbilt University, Nashville, TN, USA
}

\begin{abstract}
Emotional stimuli tend to capture and hold attention more than non-emotional stimuli do. Aversive pictures have been found to impair perception of visual targets even after the emotional information has disappeared. The benefits of such interlinked emotion and attention systems have sometimes been discussed within an evolutionary framework, with a survival advantage attributed to early detection of threatening stimuli. However, consistent with recent suggestions that attention is drawn to arousing stimuli regardless of whether they are positive or negative, the current investigation found that erotic distractors - generally rated as both pleasing and arousing - consistently elicited a transient "emotion-induced blindness" similar to that caused by aversive distractors (Experiment 1). This effect persisted despite performance-based monetary incentives to ignore the distractors (Experiment 2), and following attentional manipulations that reduced interference from aversive images (Experiment 3). The findings indicate that positively arousing stimuli can spontaneously cause emotion-induced deficits in visual processing, just as aversive stimuli can.
\end{abstract}

The visual world is bursting with information, and stimuli continually compete for a perceiver's attention: stimuli that win often reach awareness, whereas those that lose frequently go unnoticed (Mack \& Rock, 1998; Most, Simons, Scholl, Jimenez, Clifford, \& Chabris, 2001). Emotionally salient stimuli seem particularly advantaged in their ability to capture and hold attention (Anderson \& Phelps, 2001; Barnard, Ramponi, Battye, \& Mackintosh, 2005; Fox, Russo, Bowles, \& Dutton, 2001; Most, Chun, Widders, \& Zald, 2005; Öhman, Flykt, \& Esteves, 2001a). This may be with

Correspondence should be addressed to: Steven B. Most, Department of Psychology, 108 Wolf Hall, University of Delaware, Newark, DE 19716-2577, USA.

E-mail: most@psych.udel.edu

(C) 2007 Psychology Press, an imprint of the Taylor \& Francis Group, an Informa business www.psypress.com/cogemotion

DOI: $10.1080 / 02699930600959340$ 
good reason: if something in the environment is threatening, then survival can be maximised by interlinked emotion and attention systems that ensure that this information is immediately prioritised. Indeed, based partly on evidence that threatening cues do appear to capture attention, cases have been made for evolutionarily developed pre-attentive threat-detection modules (Öhman et al., 2001a; Pratto \& John, 1991; but see Batty, Cave, \& Pauli, 2005). Yet, the specificity of these phenomena to negativity is questionable. A disproportionate number of studies in the area have tested emotionally negative stimuli, but positive arousing stimuli may produce similar effects on attention. Indeed, some studies suggest that emotionally arousing stimuli can capture attention in general, regardless of whether they are considered positive or negative (Anderson, 2005; Arnell, Killman, \& Fijavz, in press; Schimmack, 2005).

Studies incorporating both emotionally appealing and emotionally aversive stimuli have tended to find an asymmetry, with appealing stimuli modulating attention to a lesser degree than aversive ones (e.g., Pratto \& John, 1991). However, most positive stimuli tend to be rated as less intensely arousing than negative stimuli (see, for example, Lang, Bradley, \& Cuthbert, 2001). Erotic stimuli are an exception - they generally are rated as both emotionally positive and highly arousing by both men and women (Bradley, Codispoti, Cuthbert, \& Lang, 2001a; Lang et al., 2001). In some cases, erotic stimuli have been found to affect attention similarly to arousing negative stimuli, supporting the hypothesis that arousal is probably more important than valence (i.e., positive vs. negative) or specific threat features in the emotional modulation of attention (Anderson, 2005; Schimmack, 2005; Schupp, Junghofer, Weike, \& Hamm, 2004).

Recently, it has been reported that aversive pictures capture and hold attention to such a degree that they interfere with the detection of targets even after the aversive picture has disappeared (Most et al., 2005). In this paradigm, participants searched for a target image embedded within a rapid stream of pictures. The participants were substantially worse at detecting the target when it followed an emotionally negative distractor relative to an emotionally neutral one, an effect operationalised as "emotion-induced blindness", or "attentional rubbernecking" (Most et al., 2005). Similar effects were observed when the emotionality of the distractor had been established through aversive conditioning (Smith, Most, Newsome, \& Zald, 2006). In both cases, the emotionally negative pictures captured and held attention enough to induce a spontaneous "attentional blink" (i.e., the failure to see targets appearing very soon after a previously attended target; Chun \& Potter, 1995; Raymond, Shapiro, \& Arnell, 1992). Similar, but smaller, spontaneous effects have been observed in paradigms employing words instead of pictures (Arnell et al., in press; Barnard et al., 2005). The current series of experiments test the degree to which positively arousing 
distractors pre-empt the processing of goal-related non-emotional targets. We specifically hypothesised that erotic stimuli would induce spontaneous attentional blinks similar to those seen following aversive pictures.

\section{EXPERIMENT 1}

Although men and women both tend to rate erotic stimuli as visually pleasing, men do so to a greater degree than women and also show signs of greater physiological reactivity to them (e.g., Bradley, Codispoti, Sabatinelli, \& Lang., 2001b). Therefore, in Experiment 1, we tested only males. Each emotional distractor was an erotically charged picture of a female (mostly nudes), and accuracy following these distractors was compared to accuracy following pictures of fully clothed females and of fully clothed males.

\section{Method}

Participants. Participants were 20 males from the Vanderbilt community, each of whom gave informed written consent before the study began, as per Vanderbilt University Institutional Review Board (IRB) guidelines.

Materials and procedure. Stimuli were colour photographs-56 of female nudes, 56 of clothed females, 56 of clothed males, 252 of upright landscape/architectural scenes, and 168 of target images (84 landscape/ architectural photos rotated 90 degrees to the left and right). Stimuli were $12.4 \mathrm{~cm}$ wide, $9.3 \mathrm{~cm}$ high, and were presented on a CRT monitor with a $75 \mathrm{~Hz}$ refresh rate via the Psychophysics Toolbox extensions for Matlab (Brainard, 1997; Pelli, 1997). In order to verify that the erotic pictures were viewed positively, eight additional males rated the pictures for valence and arousal on a scale of 1-9 (1=very negativelvery unstimulating; $9=$ very positivelvery arousing). To maximise the representativeness of this sample, ratings for each category were screened for outliers; if a rater was more than two standard deviations away from the mean in any category, his ratings for all categories were removed from the analyses. One male who rated the pictures of clothed males as being exceptionally unpleasant (valence $=1.06$ ) was removed. Results verified that the pictures of nude females were rated as more positive $(M=7.1, S D=0.9)$ and more arousing $(M=6.1, S D=1.1)$ than either the clothed males (valence: $M=4.6, S D=0.6$; arousal: $M=1.5$, $S D=0.5$ ) or the clothed females (valence: $M=5.6, S D=0.3$; arousal: $M=3.0, S D=1.4$ ), all $p$ s $\leq .006$.

Experimental trials consisted of a rapid serial visual presentation (RSVP) stream of 17 images, each presented for $106 \mathrm{~ms}$ (see Figure 1). Except for two images, all were upright landscape/architectural photographs; the remaining two images consisted of the critical distractor and the target stimulus. 


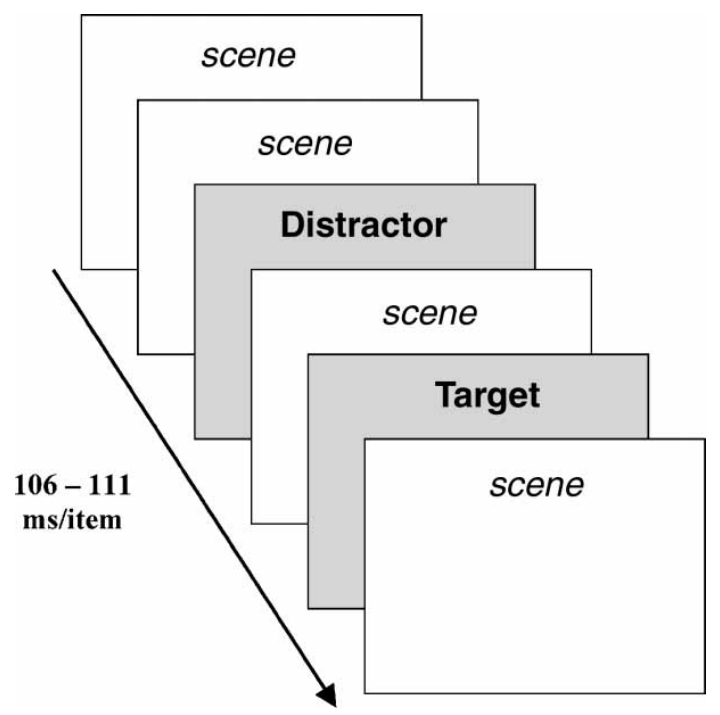

Figure 1. Schematic diagram of part of a trial. Distractors were either neutral or emotional pictures, and targets were landscape/architectural photos that had been rotated 90 degrees clockwise or counter-clockwise. On each trial, participants indicated which way the target had been rotated. Distractors and targets were embedded in rapid streams of scenes (upright landscape/architectural photos). Experiments 1, 2, and 3 varied in the duration of each picture presentation (106-111 ms/ picture).

Depending on the trial, the 4th, 6th, or 8th stimulus was the critical distractor: either erotically charged pictures of females (nude or in revealing lingerie), clothed females, or clothed males. ${ }^{1}$ The target stimulus consisted of a landscape/architectural photo rotated 90 degrees to the left or right, which appeared either 2 or 8 items after the critical distractor ( $\operatorname{Lag} 2$ and Lag 8, respectively; see Figure 1). At the end of each trial, participants pressed either the left arrow key or the right arrow key to indicate the direction in which the target had been rotated.

\footnotetext{
${ }^{1}$ The complexity of all critical distractors in this experiment was limited by the fact that each distractor depicted only one or two people. Although we did not explicitly match for low-level features such as luminance or colour, our previous report on responses to negative stimuli incorporated scrambled pictures and demonstrated that emotional effects within this paradigm cannot be attributed to such low-level features (Most et al., 2005). This appears to be the case here as well: for example, pictures of nude females impaired target perception far more than did pictures of clothed females even though the average luminance of the clothed females was greater, as assessed by their mean luminance value using the histogram function in Adobe Photoshop CS (Adobe Systems Inc., San Jose, CA). In contrast, the nude pictures in Experiments 2 and 3 had greater mean luminosity than the neutral pictures, but they again caused a larger attentional blink. Taken together, the evidence suggests no systematic relationship between such low-level features and the emotion-induced effect.
} 
Participants sat at a comfortable distance of about 24 inches from the screen and first completed a 16-trial practice session containing no critical distractors or pictures from the actual experiment. Then, without the experimenter or others present, they completed 6 blocks of 28 experimental trials. The instructions to the experimental trials emphasised that the rotated target would always be a landscape/architectural photo, and that participants should ignore all pictures of people. The computer randomised the order of the trials, as well as which pictures were paired with which Lag.

\section{Results and discussion}

Erotically charged stimuli elicited an attentional blink even though they were never the targets of a search and participants knew to ignore all pictures of people. An omnibus ANOVA revealed significant main effects of distractor Category, $F(2,38)=20.31, p<.001$, and of $\operatorname{Lag}, F(1,19)=80.26, p<.001$, as well as a Category $\times$ Lag interaction, $F(2,38)=17.35, p<.001$ (see Figure 2). By Lag 8, participants had recovered to $89 \%$ accuracy or better in all conditions, and an ANOVA performed on accuracy at Lag 8 revealed no difference between Categories there, $F(2,38)=1.01, p=.373$ (Clothed Male: $M=91 \%, S D=7 \%$; Clothed Female: $M=91 \%, S D=7 \%$; Erotic Female: $M=89 \%, S D=10 \%)$. In contrast, a large effect of Category emerged at Lag 2, $F(2,38)=29.15, p<.001$ (Clothed Male: $M=83 \%, S D=12 \%$; Clothed Female: $M=85 \%, S D=11 \%$; Erotic Female: $M=66 \%, S D=10 \%$ ). Thus,

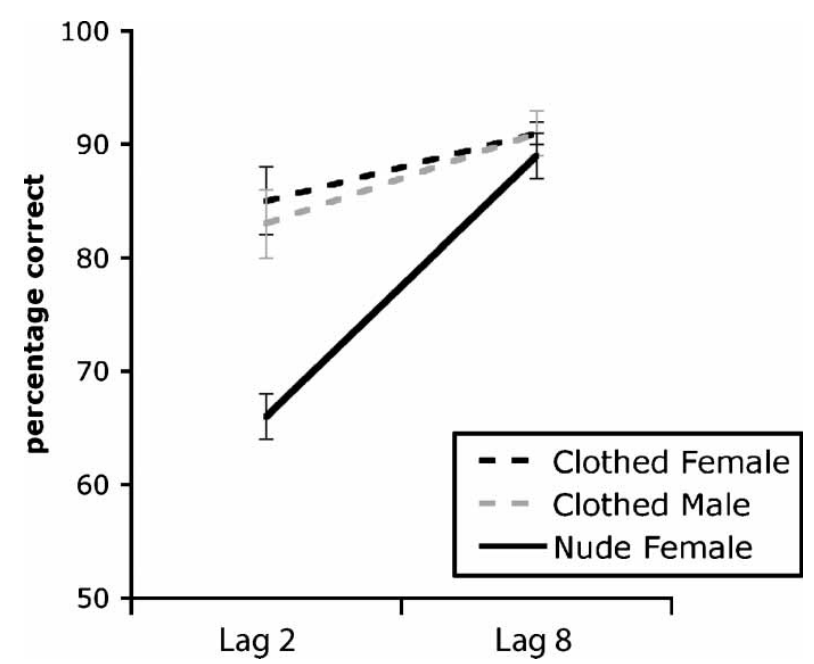

Figure 2. Results from Experiment 1. At Lag 2, participants were significantly worse at reporting targets when they followed pictures of nude females than when they followed pictures of clothed males or females. By Lag 8, participants had recovered to an accuracy rate of $89 \%$ or above regardless of distractor. 
consistent with previous reports (Most et al., 2005), we limited the remainder of our analyses to Lag 2 . In this and subsequent experiments, $t$-tests were planned and are reported 2-tailed.

At Lag 2, accuracy in reporting a target following an image of a clothed female was no different than when following an image of a clothed male, $t(19)=0.98, p=.34$. In contrast, accuracy was significantly worse following an erotic image of a female, both in comparison to clothed males, $t(19)=$ $6.18, p<.001$, and to clothed females, $t(19)=6.11, p<.001$.

Thus, although participants knew pictures of people to be task irrelevant, when the pictures were erotically charged, attention appeared to have been held enough to induce temporary impairments in processing subsequent visual targets relative to when the pictures were of clothed males or clothed females. Notably, performance was worse at Lag 2 than Lag 8 for all distractor types, indicating that some general distraction was caused by the novel appearance of people within the stream of landscapes and buildings. The critical aspect of these results, however, is the difference in performance between erotic and non-erotic conditions.

Of course, it is possible that participants were not distracted from their search against their will. With no incentive to do otherwise, they may have actively looked for the erotic images as well as for the targets. If so, then the effect demonstrated here should be interpreted no differently than the standard attentional blink (Chun \& Potter, 1995; Raymond et al., 1992), where volitional attention to a first target impairs detection of a rapidly succeeding second target. In Experiment 2, we addressed this issue by including a monetary incentive for people to ignore the emotional distractors.

\section{EXPERIMENT 2}

To help counteract the possibility that participants might actively search for an erotic distractor as well as for the target, we introduced a large performance-based monetary reward in order to provide incentive for them to exclusively focus on target detection. Participants completed three "Reward" and three "Non-Reward" blocks of trials. On Reward blocks, participants were informed that their performance levels at detecting the target could lead to greater monetary remuneration (up to \$90). On NonReward blocks, participants were informed that their performance did not affect the level of remuneration. Participants were aware of which blocks were which, and were told to try their best to detect the actual target regardless of condition. In an additional change from Experiment 1, the "clothed male" and "clothed female" sets of pictures were replaced by a set of neutral pictures drawn mostly from the International Affective Picture System (IAPS; Lang et al., 2001) and by a set of erotic pictures of 
male-female couples. The latter was done with an eye towards Experiment 3 , in which both male and female participants were to be tested; it was important to ensure that the increased visual complexity of having two people depicted in a distractor did not diminish the effect relative to when only one person was depicted (i.e., most pictures in the erotic female condition depicted only one person).

\section{Method}

Participants. Participants were 20 males, ages 18-28, each of whom gave informed written consent before the study began, as per Vanderbilt University Institutional Review Board (IRB) guidelines. Participants were compensated at a base rate of $\$ 15$, with the understanding that they could increase this amount depending on their performance.

Materials and procedure. The pictorial stimuli consisted of the same landscape/architectural, female nude, and rotated target images as Experiment 1 . The notable differences in stimuli between studies were that the "clothed male" and "clothed female" sets of pictures were replaced with a set of neutral pictures (people and animals) that we previously used in a similar experiment (Most et al., 2005) and with a set of pictures of erotic malefemale couples. Both of these new sets were drawn partly from the IAPS (Lang et al., 2001) and were supplemented with pictures of similar content from publicly available sources. All pictures were $11.2 \times 8.4 \mathrm{~cm}$ in size and presented on a CRT monitor with a $72 \mathrm{~Hz}$ refresh rate, yielding a presentation rate of $111 \mathrm{~ms} /$ picture.

The experimental blocks of 28 trials were divided into three "Reward" blocks and three "No Reward" blocks. The type of blocks alternated (with half the participants receiving the Reward condition first), and each block began with a screen indicating whether it was a Reward or No Reward block. Across the Reward condition, participants earned an extra $\$ 10$ if overall accuracy was greater than $80 \%$ and an extra $\$ 25$ ( $\$ 40$ total) if overall accuracy was greater than $90 \%$. Additionally, the participant with the highest overall accuracy in the Reward condition received a $\$ 50$ bonus at the end of the study ( $\$ 90$ total), and participants were informed of this prior to the experiment. For the No Reward condition, participants were informed that performance would not affect monetary compensation, but that they should continue to focus and perform as accurately as possible. To allow participants to gauge their own performance during the task, each trialregardless of condition - contained auditory feedback: a "bell" sound played for each correct response, and an "alert" sound played for each incorrect response. At the end of the experiment, participants indicated on $1-5$ scales the degree to which they had tried to see the rotated targets and the degree to which they had tried to see the erotic distractors $(1=$ not at all; 
$5=$ as much as possible), as well as the relative degree to which they had concentrated on each $(1=$ trying exclusively to see erotic pictures; $5=$ trying exclusively to perform well).

With an eye towards potential future studies involving individual differences (which would require larger sample sizes), participants were asked to complete three personality-related surveys (Harm Avoidance, Cloninger, Przybeck, \& Svrakic, 1991; Neuroticism, Costa \& McCrae, 1992; and an Attentional Control scale, Derryberry \& Reed, 2002). However, these measures were administered for piloting purposes only; they are not directly relevant to the current experiments and therefore are not reported further.

\section{Results and discussion}

An omnibus 2 (Reward: money vs. no money) $\times 3$ (distractor Category: nude couples vs. nude females vs. neutral pictures) $\times 2$ (Lag: Lag 2 vs. lag 8) ANOVA revealed main effects of Reward, $F(1,19)=5.08, p=.036$, Category, $F(2,38)=18.74, p<.001$, and Lag, $F(1,19)=60.06, p<.001$, as well as an interaction between Category and Lag, $F(2,38)=4.43$, $p=.019$.

A 2 (Reward) $\times 3$ (Category) ANOVA at Lag 8 revealed a main effect of distractor Category, $F(2,38)=5.95, p=.006$, indicating that even by Lag 8 accuracy in the two erotic conditions did not always recover to the same level as that in the neutral condition. However, all accuracies at Lag 8 were above $90 \%$ for both the Reward condition (neutral: $M=97 \%, S D=5 \%$; nude females: $M=92 \%, S D=8 \%$; erotic couples: $M=93 \%, S D=6 \%$ ) and the No Reward condition (neutral: $M=96 \% S D=5 \%$; nude females: $M=91 \%$, $S D=9 \%$; erotic couples: $M=95 \%, S D=7 \%$ ). Therefore, as in Experiment 1, we limit the remainder of our analyses to Lag 2 .

At Lag 2, a 2 (Reward) $\times 3$ (Category) ANOVA revealed a weak main effect of Reward, $F(1,19)=4.64, p=.044$, and a main effect of Category, $F(2,38)=12.14, p<.001$, but no interaction between the two factors, $F(2,38)=0.35, p>.50$. However, planned $t$-tests revealed that when the distractor categories were tested separately, the weak main effect of Reward disappeared for neutral distractors [no reward: $M=84 \%, S D=16 \%$; reward: $M=89 \%, S D=8 \% ; t(19)=1.51, p=.147$ ], single nudes [no reward: $M=$ $68 \%, S D=18 \%$; reward: $M=76 \%, S D=18 \% ; t(19)=1.62, p=.120]$, and nude couples [no reward: $M=75 \%, S D=17 \%$; reward: $M=77 \%, S D=13 \%$; $t(19)=0.58, p>.50]$.

To assess the degree to which target perception was impaired in the erotic conditions relative to the neutral condition (i.e., an emotion-specific deficit), the data were submitted to two separate ANOVAs. A 2 (Reward) $\times 2$ 
(Category: neutral vs. nude couples) ANOVA revealed that inability to report the target was greater following the nude couples than the neutral distractors, $F(1,19)=14.54, p=.001$. There was no Reward $\times$ Category interaction, suggesting that the relative decrement in performance did not change with the prospect of reward $(p>.60)$. A second 2 (Reward) $\times 2$ (Category: neutral vs. nude females) ANOVA revealed that ability to report the target was worse following pictures of nude females than following neutral pictures, $F(1,19)=18.06, p<.001$. Again, the lack of an interaction suggested that this relative decrement did not change as a function of reward $(p>.60)$. Finally, an additional 2 (Reward) $\times 2$ (Category: nude females vs. nude couples) ANOVA revealed neither a main effect of Category, $F(1,19)=$ $1.76, p=.20$, nor any Category $\times$ Reward interaction $(p>.45)$.

All participants circled " 5 " on a 5-point scale to indicate that they had tried to perform as well as possible on the task. All but two also circled " 5 " on a 5 -point scale to indicate that they had tried as much as possible to see the rotated target on each trial; two participants circled "4". There was more variability in people's ratings of how hard they had tried to see the erotic pictures, ranging from 1 to $4(M=1.81, S D=0.91)$. However, these ratings correlated neither with the difference in distraction caused by nude females vs. neutral pictures or by erotic couples vs. neutral pictures, nor with the influence of reward on these differences ( $p s>.45)$; thus, the two erotic conditions did not differ significantly from each other in their effects.

Despite the prospect of a substantial monetary reward, participants were not able to ignore the erotic distractors, which temporarily impaired subsequent target detection. With the monetary incentive at stake, it seems unlikely that participants' poorer performance during erotic trials simply resulted from their not trying to focus attention on the primary target detection task. Participants own ratings of how hard they had tried to see the targets also suggest that they were truly engaged in the primary task. However, simply trying hard might not be enough. In Experiment 3, we examined whether ignoring of the erotic distractors might be improved by a qualitative change in attentional strategy, or attentional set. For example, an earlier study demonstrated that specific knowledge of the target (e.g., the rotated image will be a building) improved some participants' ability to detect the target, even when it followed emotionally negative images (Most et al., 2005). In Experiment 3, we performed a similar manipulation of participants' attentional set while continuing to offer an extra monetary reward. For this final experiment, we tested both male and female participants in order to determine whether similar erotica-induced attentional blinks occur for both genders. 


\section{EXPERIMENT 3}

In Experiment 2, it was possible that the prospect of an additional monetary reward motivated participants to try hard to ignore emotional distractors, but that they did so without qualitatively altering their attentional strategy. Previous experiments using emotionally negative distractors demonstrated that, in some people, narrowing the range of features involved in a search helped them to ignore the distractors. This makes sense intuitively: if you search for a friend in a crowd, you will be less likely to attend mistakenly to other people if you know specifically what your friend is wearing. In the context of the current experiment, we manipulated the specificity of the participants' search by informing them that their rotated target would be a picture either of a building (specific attentional set condition) or of a building or landscape (nonspecific attentional set condition). In actuality, only trials containing buildings as targets were analysed. Negative distractors were included in this experiment (in place of the nude females from Experiment 2) in order to gauge whether manipulations of attentional set would influence attentional blinks caused by erotic and negative distractors similarly. As in Experiment 2, participants were told that they could increase their monetary compensation through accurate performance.

An equal number of males and females participated, and the erotic pictures were of male-female couples (from Experiment 2). Although both men and women tend to rate erotic pictures as appealing, men tend to do so to a greater degree and show greater levels of physiological reactivity to them (Bradley et al., 2001b). Furthermore, recent evidence suggests that brain regions such as the amygdala and hypothalamus show greater activation among men than women when viewing erotic images (Hamann, Herman, Nolan, \& Wallen, 2004). Therefore, we sought to determine whether erotic pictures induced a spontaneous attentional blink of comparable magnitude in women and men.

\section{Method}

Participants. Participants were 8 males (ages 19-51) and 8 females (ages 21-33), each of whom gave informed written consent before the study began, as per Vanderbilt University Institutional Review Board (IRB) guidelines. Participants were compensated at a base rate of $\$ 15$, with the understanding that they could increase this amount depending on their performance.

Materials and procedure. The materials and procedure were identical to those in Experiment 2, with a few notable exceptions. Stimuli were $9.2 \times$ $6.9 \mathrm{~cm}$ colour photographs presented on a CRT monitor with a $75 \mathrm{~Hz}$ refresh rate for $106 \mathrm{~ms} /$ picture. Emotionally negative pictures (e.g., gore, violence, threatening animals) were used as distractors in place of the nude females condition from Experiment 2; most of these were drawn from the 
IAPS (Lang et al., 2001), and these were supplemented by pictures with similar content collected from publicly available sources (see Most et al., 2005). ${ }^{2}$ Erotic stimuli were the pictures of male-female couples used in Experiment 2.

To verify that the erotic pictures were viewed as positive and arousing, 15 additional volunteers ( 7 women) rated the erotic, negative, and a set of neutral IAPS pictures according to the same procedure described in Experiment 1 . One female rated erotic pictures as exceptionally negative (valence $=1.98$ ) and neutral pictures as exceptionally positive (valence $=$ 7.49); these ratings were more than two standard deviations away from those of the other female raters and this volunteer's ratings for all categories were therefore removed from the analyses. Results verified that erotic pictures were rated as more positive $(M=6.8, S D=1.1)$ than either the negative $(M=2.1, S D=0.5)$ or neutral $(M=5.1, S D=0.4)$ pictures, $p$ s $<.001$. Erotic pictures were also rated as more arousing than the neutral pictures $(M=6.8, S D=1.4$ and $M=2.3, S D=0.9$, respectively, $p<.001)$ and as inducing about the same degree of arousal as the negative pictures $(M=7.0, S D=0.9, p=.317)$. Although men and women alike rated the erotic pictures as being more arousing than neutral pictures $(p s<.001)$, men rated the erotic pictures as being substantially more pleasing than neutral pictures (erotic: $M=7.4, S D=0.8$; neutral: $M=5.1, S D=0.5 ; p<.001$ ), whereas women rated them as only slightly more pleasing (erotic: $M=6.0$, $S D=1.1$; neutral: $M=5.2, S D=0.2 ; p=.193$ ). Interestingly, men rated erotic pictures as slightly more arousing than negative pictures $(M=7.2$, $S D=1.3$ and $M=7.0, S D=1.0$, respectively, $p=.168$ ), whereas women rated erotic pictures as somewhat less arousing than negative ones $(M=6.2$, $S D=1.3$ and $M=7.1, S D=0.9$, respectively, $p=.054$ ). Although the small sample sizes here preclude firm conclusions about sex differences in ratings, this pattern is notable in its consistency with previous reports (e.g., Bradley et al., 2001a). ${ }^{3}$

As in Experiment 2, participants in Experiment 3 were told that they could increase their monetary compensation through accurate performance.

\footnotetext{
${ }^{2}$ Stimuli included 39 negative IAPS images (valence $=1.85, S D=0.43$; arousal $=6.34, S D=$ $0.63)$.

${ }^{3}$ Note that we cannot rule out the possibility that some female subjects in the main experiment had a similarly negative response to erotic pictures as the one female outlier among our raters. However, to anticipate the results, the decrement in performance was robust across subjects, such that the results could not simply reflect a response from one or two females who might have rated the erotica as unpleasant, had such participants been present in the experimental sample at all. Among the independent raters, men overwhelmingly rated erotic pictures as positive, and women generally did so as well, albeit with lesser enthusiasm. No participants in the attentional blink part of the study volunteered that they found the erotica unpleasant during debriefing.
} 
Participants whose overall accuracy exceeded $80 \%$ received an extra $\$ 10$, and those whose accuracy exceeded $90 \%$ received an extra $\$ 25$ (\$40 total). In addition, the participant with the highest overall accuracy received an additional $\$ 50$ bonus at the end of the study ( $\$ 90$ total), and participants were informed of this prospect prior to beginning the experiment. Note that in Experiment 3, all trial blocks were part of reward blocks, and no comparison with non-reward trials was performed.

The critical manipulation in this experiment was the degree of specificity with which targets were described. In two of four 70-trial blocks, the target was always a picture of a rotated building, and participants were informed of this at the start of the blocks. These blocks represented the specific attentional set condition. In the other two blocks, the target in each trial was a rotated picture of either a building or a landscape with no building, and participants were informed that it could be either. These blocks represented the nonspecific attentional set condition. In actuality, all analysed trials-regardless of condition-contained targets randomly drawn from the same pool of pictures of buildings. The specificity of participants' attentional set was manipulated (in addition to the instructions) through the inclusion of "filler trials" that were not analysed. In the specific attentional set condition, filler trials contained buildings as targets; in the nonspecific attentional set condition, they contained landscapes with no buildings as targets. Filler trials contained no critical distractors. Each block contained 14 negative, 14 neutral, 14 erotic, and 28 filler trials. The specific and nonspecific attentional set conditions were presented in alternating blocks, with half the participants receiving the specific attentional set condition first. As in Experiment 2, participants received immediate auditory feedback about their accuracy on each trial.

In order to collect pilot data for future studies involving individual differences, participants were asked to complete the three personality-related surveys used in Experiment 2, as well as an additional scale (Positive And Negative Affect Scale, Watson, Clark, \& Tellegen, 1988). However, as individual-difference effects require a larger sample size than was tested, the data are not reported here.

\section{Results and discussion}

An omnibus 2 (Lag: Lag 2 vs. Lag 8 ) $\times 3$ (distractor Category: negative vs. erotic vs. neutral) $\times 2$ (Attentional Set: specific vs. nonspecific) ANOVA revealed significant effects of Lag, $F(1,15)=45.35, p<.001$, distractor Category, $F(2,30)=8.61, p=.001$, and Attentional Set, $F(1,15)=5.67$, $p=.031$, as well as an interaction between Category and Lag, $F(2,30)=8.53$, $p=.001$. At Lag 8 , a 2 (Attentional Set) $\times 3$ (Category) ANOVA revealed no significant main effects or interactions (all $p \mathrm{~s}>.10$; all accuracies $>95 \%$ ). 
Therefore, as in Experiments 1 and 2, all subsequent analyses are limited to Lag 2.

Planned $t$-tests of Lag 2 performance revealed that adopting a specific attentional set instead of a nonspecific attentional set led to improved accuracy following a neutral distractor [nonspecific: $M=87 \%, S D=10 \%$; specific: $M=93 \%, S D=8 \% ; t(15)=2.02, p=.061$ ], and following an emotionally negative distractor [nonspecific: $M=76 \%, S D=15 \%$; specific: $M=86 \%, S D=10 \% ; t(15)=2.71, p=.016]$. Critically, attentional set did not influence target detection when the critical distractor was an erotic image [nonspecific: $M=80 \%, S D=15 \%$; specific: $M=80 \%, S D=17 \% ; t(15)=$ $0.08, p>.90$; see Figure 3]. This is despite the fact that, as in Experiment 2 , there was a large monetary incentive to perform well.

In order to further delineate the influence of attentional set and subjects' sex on the impact of emotional distractors, we conducted two separate analyses comparing negative to neutral distractors and comparing erotic to neutral distractors, both of which incorporated subjects' sex as a factor. These separate 2 (distractor Category) $\times 2$ (Attentional Set) $\times 2$ (subject Sex) ANOVAs allowed us to examine whether the impact of negative and erotic (positive), relative to neutral, distractors were differentially affected by attentional set. In an overall main effect, emotionally negative distractors, relative to neutral distractors, led to decreased accuracy in responding to a target, $F(1,14)=13.14, p=.003$. A lack of a Category $\times$ Attentional Set interaction $(p>.30)$ demonstrated that although adopting a specific attentional set had improved performance following a negative distractor, doing so did not affect the relative degree to which negative distractors impaired performance more than neutral distractors. Similarly, the main effect comparing erotic distractors to neutral ones revealed that erotic distractors led to decreased accuracy relative to neutral distractors, $F(1,14)=18.68, p=$ .001. A lack of a Category $\times$ Attentional Set interaction again showed that adopting a specific attentional set did not diminish the degree of the relative visual deficit $(p>40)$. Subjects' sex had no main effects in these tests nor interactions with other variables.

A final 2 (Category: erotic vs. negative) $\times 2$ (subject Sex) ANOVA revealed that overall accuracy did not differ between the negative and the erotic conditions, $F(1,15)=0.10, p=.752$, but - in this analysis directly comparing the effects of negative to erotic distractors-there was a significant interaction between Category and Sex, $F(1,14)=6.14, p=.027$, indicating that whereas males were somewhat more impaired overall by erotic distractors than negative ones at Lag 2 (negative: $M=81 \%, S D=12 \%$; erotic: $M=76 \%, S D=17 \%$ ), females showed somewhat the reverse pattern (negative: $M=80 \%, S D=16 \%$; erotic: $M=84 \%, S D=14 \%$ ). This pattern is consistent with the pattern of arousal ratings from our additional volunteers and with previous research demonstrating that men tend rate erotic pictures 

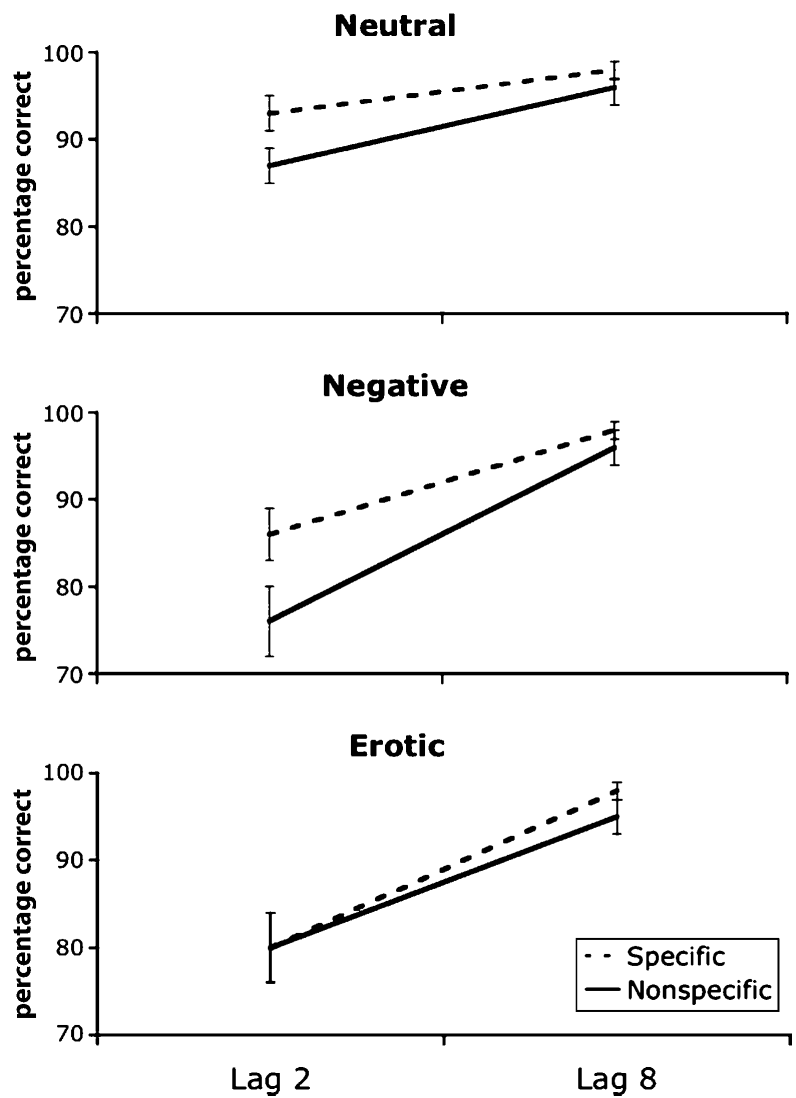

Figure 3. Results from Experiment 3, showing the effects of attentional set for neutral, negative and erotic trials. When participants adopted a specific attentional set (i.e., look for a rotated building), they were better able to ignore neutral and negative distractors at Lag 2 (indexed through better target detection) than when they adopted a nonspecific attentional set (i.e., look for a rotated building or landscape). This improvement occurred even though only trials containing buildings as targets were analysed. In contrast, adopting a specific attentional set did not improve performance following an erotic distractor.

as more arousing and show more physiological reactivity to erotica than women do, whereas women tend to rate negative pictures as more arousing and show greater physiological reactivity to negative images than men do (Bradley et al., 2001a).

\section{GENERAL DISCUSSION}

Prominent theories suggest that effects of emotion on attention are specific to threat-relatedness or negativity (e.g., Koster, Crombez, Van Damme, 
Verschuere, \& De Houwer, 2004; Öhman et al., 2001a; Öhman, Lundqvist, \& Esteves, 2001b; Pratto \& John, 1991). This assumption might make sense from an evolutionary perspective - a perceptual system that pre-empts nonemotional information in order to prioritise potential threat would have substantial survival value. However, a recent spate of research has instead suggested that emotional effects on attention stem from emotional arousal, independent of whether something is considered positive or negative (Anderson, 2005; Schimmack, 2005). Consistent with the latter hypothesis, we found that when participants searched for a target embedded within a rapid stream of pictures, task-irrelevant, positively arousing emotional distractors captured and held attention to such a degree that they induced brief deficits in processing the subsequent target. This phenomenon, termed "emotion-induced blindness" or "attentional rubbernecking", has previously been demonstrated using emotionally negative distractors (Most et al., 2005).

The weak effects of emotionally positive stimuli on attention found in some previous studies may be due to the fact that most positively valenced stimuli tend not to be as strongly arousing as negatively valenced stimuli (see, for example, Lang et al., 2001). Erotic images are an exception; they tend to be experienced as both pleasing and arousing by both men and women (Bradley et al., 2001b; Lang et al., 2001). Of course, it is possible that prioritisation of erotic information has evolutionary value (e.g., signalling a potential mating opportunity), leading to its ability to capture attention. It is unclear whether the present results would generalise to equally arousing non-erotic positive stimuli. For example, attractive faces have previously been found to affect attentional performance in other paradigms (e.g., Schimmack, 2005). Nevertheless, the data make clear that emotional inducers of spontaneous attentional blinks are not limited to negatively valenced or threat-related stimuli.

Indeed, the present data suggest that the attentional blink induced by erotic images may be even less amenable to modulation by attentional set than the blink caused by negative images. As in previous research (Most et al., 2005), when people adopted a specific attentional set rather than a nonspecific one, they were less likely to be distracted by a task-irrelevant negative distractor. This was not the case in the erotic condition. Thus, in this particular instance, the effects caused by erotica appeared even more robust than those caused by negative images. While there is clearly a similarity to the emotion-induced blindness evoked by aversive and erotic images, the differential effects of attentional set indicate that these two classes of stimuli may not be processed in an identical fashion.

Individual differences in the size of the blink emerged as a function of participants' sex. In Experiment 3, men showed a slightly greater sensitivity to erotic than to aversive stimuli, whereas women showed a slightly greater blink in response to the aversive than to erotic images. This is made all the 
more intriguing by the fact that among a small group of additional volunteers, men rated erotic pictures as more arousing than negative ones, whereas women showed the opposite tendency. Although the small size of this group alone precludes firm conclusions about sex differences in ratings, these patterns notably mirror those described in previous reports, which additionally described similar patterns emerging from physiological indices of arousal (e.g., Bradley et al., 2001b). Taken together, such patterns lend credence to the notion that emotional arousal, per se, plays a primary role in inducing a spontaneous attentional blink (see also Arnell et al., in press).

In addition to possible physiological determinants of this effect, it is also worth considering cultural factors that might lead to individual differences in the magnitude of the blink. For example, in cultures where partial exposure of one's body is considered taboo, one might predict a greater degree of emotion-induced blindness than seen in the present study. In contrast, in societies with more liberal approaches to nudity and sex, one might predict a weaker effect. Such a finding might suggest that the eroticainduced blink is influenced by learning - for example, either a learned taboo or a lack of learned adaptation to nudity - rather than representing an immutable, evolutionarily imbued tendency.

One concern in interpreting the current findings is that participants might have intentionally tried to see the erotic pictures. We addressed this in Experiments 2 and 3, where participants had a significant financial stake in performing well. Thus, to the extent that participants tried to see a fleeting erotic image, they did this despite a substantial incentive to perform well on the task. When questioned, participants indicated that they had tried hard on the task, and most, but not all, reported that they did not try to look for the erotic images. Importantly, there was no association between subject's report of the extent that they tried to look for the erotic images and actual performance on the task. The strongest interpretation of this data is that erotic distractors capture attention and impair subsequent perception automatically. The weakest interpretation is no less striking: to the degree that participants actively looked for the erotic pictures at some level, they did so even in the face of a large monetary incentive to do otherwise, and this urge led to a brief, involuntary impairment of subsequent visual processing.

It is tempting to extrapolate from these results implications for everyday life. For example, it is an adage of the advertising age that "sex sells", and graphic billboards and signs are sometimes prominent on roadways and other venues where even momentary lapses of perception could be dangerous. We note, however, that further research must determine the degree to which these laboratory-based effects extend to the real world. For example, the images used in these experiments were more graphic than content usually found in public (at least, for example, in the United States). Additionally, while it is likely that that emotion-induced blindness is a 
common phenomenon, we note that it is quite transient and should be distinguished from prolonged, more voluntarily attention to emotional information. Emotional distractors are common in everyday life, whether they are severe car accidents on the side of the road or erotically charged advertisements; given the current data, in situations requiring full attention, it might often be wise to anticipate and compensate for the potential occurrence of emotion-induced blindness by leaving room for error.

Manuscript received 13 December 2005

Revised manuscript received 13 July 2006 Manuscript accepted 7 August 2006

\section{REFERENCES}

Anderson, A. K. (2005). Affective influences on the attentional dynamics supporting awareness. Journal of Experimental Psychology: General, 134, 258-281.

Anderson, A. K., \& Phelps, E. A. (2001, May 17). Lesions of the human amygdala impair enhanced perception of emotionally salient events. Nature, 411, 305-309.

Arnell, K. M., Killman, K., \& Fijavz, D. (in press). Blinded by emotions: Target misses follow attentional capture by arousing distractors in RSVP. Emotion.

Barnard, P. J., Ramponi, C., Battye, G., \& Mackintosh, B. (2005). Anxiety and the deployment of visual attention over time. Visual Cognition, 12, 181-211.

Batty, M. J., Cave, K. R., \& Pauli, P. (2005). Abstract stimuli associated with threat through conditioning cannot be detected preattentively. Emotion, 5, 418-430.

Bradley, M. M., Codispoti, M., Cuthbert, B. N., \& Lang, P. J. (2001a). Emotion and motivation I: Defensive and appetitive reactions in picture processing. Emotion, 1, 276-298.

Bradley, M. M., Codispoti, M., Sabatinelli, D., \& Lang, P. J. (2001b). Emotion and motivation II: Sex differences in picture processing. Emotion, 1, 300-319.

Brainard, D. H. (1997). The Psychophysics Toolbox. Spatial Vision, 10, 433-436.

Chun, M. M., \& Potter, M. C. (1995). A two-stage model for multiple target detection in rapid serial visual presentation. Journal of Experimental Psychology: Human Perception and Performance, 21, 109-127.

Cloninger, R. C., Przybeck, T. R., \& Svrakic, D. M. (1991). The tridimensional personality questionnaire: US normative data. Psychological Reports, 69, 1047-1057.

Costa, P. T., Jr., \& McCrae, R. R. (1992). Revised NEO Personality Inventory and Five-Factor Inventory Professional Manual. Odessa, FL: Psychological Assessment Resources.

Derryberry, D., \& Reed, M. A. (2002). Anxiety-related attentional biases and their regulation by attentional control. Journal of Abnormal Psychology, 111, 225-236.

Fox, E., Russo, R., Bowles, R., \& Dutton, K. (2001). Do threatening stimuli draw or hold visual attention in subclinical anxiety? Journal of Experimental Psychology: General, 130, 681-700.

Hamann, S., Herman, R. A., Nolan, C. L., \& Wallen, K. (2004). Men and women differ in amygdala response to visual sexual stimuli. Nature Neuroscience, 7, 411-416.

Koster, E. H. W., Crombez, G., Van Damme, S., Verschuere, B., \& De Houwer, J. (2004). Does imminent threat capture and hold attention? Emotion, 4, 312-317.

Lang, P. J., Bradley, M. M., \& Cuthbert, B. N. (2001). International affective picture system (IAPS): Instruction manual and affective ratings. Technical Report A-5, the Center for Research in Psychophysiology, University of Florida.

Mack, A., \& Rock, I. (1998). Inattentional blindness. Cambridge, MA: MIT Press. 
Most, S. B., Chun, M. M., Widders, D. M., \& Zald, D. H. (2005). Attentional rubbernecking: Cognitive control and personality in emotion-induced blindness. Psychonomic Bulletin \& Review, 12, 654-661.

Most, S. B., Simons, D. J., Scholl, B. J., Jimenez, R., Clifford, E., \& Chabris, C. F. (2001). How not to be seen: The contribution of similarity and selective ignoring to sustained inattentional blindness. Psychological Science, 12, 9-17.

Öhman, A., Flykt, A., \& Esteves, F. (2001a). Emotion drives attention: Detecting the snake in the grass. Journal of Experimental Psychology: General, 130, 466-478.

Öhman, A., Lundqvist, D., \& Esteves, F. (2001b). The face in the crowd revisited: A threat advantage with schematic stimuli. Journal of Personality and Social Psychology, 3, 381-396.

Pelli, D. G. (1997). The VideoToolbox software for visual psychophysics: Transforming numbers into movies. Spatial Vision, 10, 437-442.

Pratto, F., \& John, O. P. (1991). Automatic vigilance: The attention-grabbing power of negative social information. Journal of Personality and Social Psychology, 61, 380-391.

Raymond, J. E., Shapiro, K. L., \& Arnell, K. M. (1992). Temporary suppression of visual processing in an RSVP task: An attentional blink? Journal of Experimental Psychology: Human Perception and Performance, 18, 849-860.

Schimmack, U. (2005). Attentional interference effects of emotional pictures: Threat, negativity, or arousal? Emotion, 5, 55-66.

Schupp, H. T., Junghofer, M., Weike, A. I., \& Hamm, A. O. (2004). The selective processing of briefly presented affective pictures: An ERP analysis. Psychophysiology, 41, 441-449.

Smith, S. D., Most, S. B., Newsome, L. A., \& Zald, D. H. (2006). An "emotional blink" of attention elicited by aversively conditioned stimuli. Emotion, 6, 523-527.

Watson, D., Clark, L., \& Tellegen, A. (1988). Development and validation of brief measures of positive and negative affect: The PANAS scales. Journal of Personality and Social Psychology, $54,1063-1070$. 